\title{
Effect of confinement on DNA dynamics in microfluidic devices
}

\author{
Richard M. Jendrejack \\ Department of Chemical Engineering, University of Wisconsin-Madison, Madison, Wisconsin 53706 \\ David C. Schwartz \\ Laboratory for Molecular and Computational Genomics, Department of Chemistry and Laboratory of \\ Genetics, University of Wisconsin-Madison, Madison, Wisconsin 53706 \\ Michael D. Graham ${ }^{\text {a) }}$ and Juan J. de Pablob) \\ Department of Chemical Engineering, University of Wisconsin-Madison, Madison, Wisconsin 53706
}

(Received 21 October 2002; accepted 17 March 2003)

\begin{abstract}
The dynamics of dissolved long-chain macromolecules are different in highly confined environments than in bulk solution. A computational method is presented here for detailed prediction of these dynamics, and applied to the behavior of $\sim 1-100 \mu \mathrm{m}$ DNA in micron-scale channels. The method is comprised of a self-consistent coarse-grained Langevin description of the polymer dynamics and a numerical solution of the flow generated by the motion of polymer segments. Diffusivity and longest relaxation time show a broad crossover from free-solution to confined behavior centered about the point $H \approx 10 S_{b}$, where $H$ is the channel width and $S_{b}$ is the free-solution chain radius of gyration. In large channels, the diffusivity is similar to that of a sphere diffusing along the centerline of a pore. For highly confined chains $\left(H / S_{b} \ll 1\right)$, Rouse-type molecular weight scaling is observed for both translational diffusivity and longest relaxation time. In the highly confined region, the scaling of equilibrium length and relaxation time with $H / S_{b}$ are in good agreement with scaling theories. In agreement with the results of Harden and Doi [J. Phys. Chem. 96, 4046 (1992)], we find that the diffusivity of highly confined chains does not follow the scaling relation predicted by Brochard and de Gennes [J. Chem. Phys. 67, 52 (1977)]; that relationship does not account for the interaction between chain and wall. (C) 2003 American Institute of Physics. [DOI: 10.1063/1.1575200]
\end{abstract}

\section{INTRODUCTION}

The behavior of a dissolved polymer chain in a confined space is central to many natural and technological processes, and has taken on renewed importance because of new and developing technologies for single molecule manipulation and analysis of DNA. ${ }^{1-7}$ For instance, in certain implementations of exonucleolytic sequence analysis it is desirable to link an individual long DNA strand to a surface (e.g., a bead) without manual intervention. ${ }^{6,7}$ The ability to engineer such tasks will be greatly improved by predictive computational tools for polymer chains in microfluidic geometries. In this work, we present a general method for dynamic simulations of macromolecules in confined geometries.

The equilibrium properties and conformations adopted by a confined chain in solution have been studied extensively. ${ }^{8} 9$ Much less theoretical or computational work is available on the dynamics of confined chains, either in equilibrium or flow, in spite of their practical importance. ${ }^{10-15}$ Qualitatively, one might envision two effects of confinement on the dynamics of a dissolved chain. First, the change in equilibrium conformation brought on by confinement may change the molecular motion (see Fig. 1). Second, and more important, as we shall see, the solvent flow in the confining geometry will also influence the poly-

\footnotetext{
${ }^{a)}$ Electronic mail: graham@engr.wisc.edu

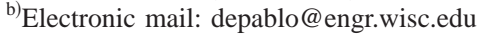

mer segment dynamics. More precisely, the mobility of an individual segment decreases while hydrodynamic interactions between segments are screened, leading to Rouse rather than Zimm scaling of the diffusivity for small channels. ${ }^{16}$ The resulting dependence of diffusivity on channel width has been predicted from scaling arguments by Brochard and de Gennes ${ }^{16}$ and from self-consistent mean field theory and the Kirkwood approximation by Harden and Doi. ${ }^{17}$ Both studies predict power law behavior of the diffusivity in terms of channel width, but the predicted exponents are different. The results of those two studies will be discussed below in more detail.

Progress beyond scaling or quasistatic results has been slow. In principle, the time scales of interest in many cases are accessible to coarse-grained, or Brownian models. ${ }^{18,19}$ Existing simulations ${ }^{20}$ of chain models in confined geometries have not accounted for changes in the hydrodynamics brought on by confinement. The central challenge is to consider polymer and solvent motions simultaneously, with the solvent motion satisfying the no-slip boundary condition on the surfaces of the confining geometry. In this work, we address this challenge with an approach that combines techniques from computational fluid dynamics and polymer physics. This method is then used to generate computational predictions of the dynamics of long DNA molecules in a channel of micrometer dimensions. Our results represent the first self-consistent Brownian dynamics treatment of polymer 


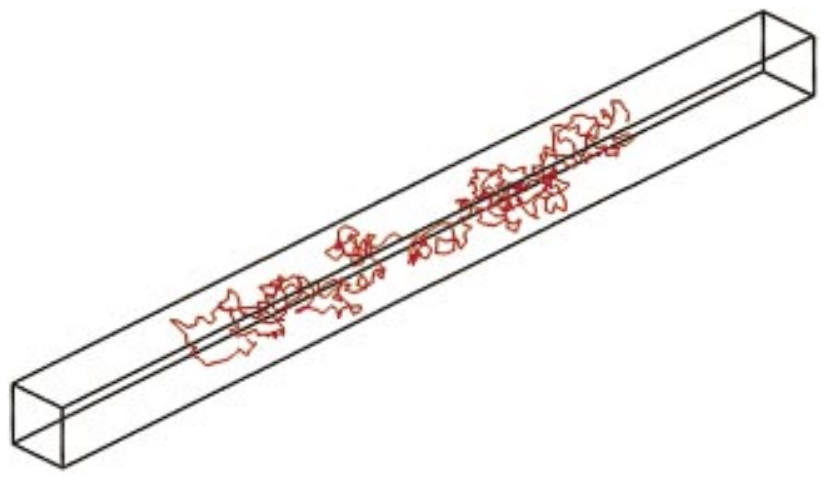

FIG. 1. (Color) A chain of 7920 Kuhn segments (bulk radius of gyration $=36.3$ Kuhn lengths) confined to a channel 30 Kuhn lengths wide.

dynamics and hydrodynamics under confinement. We find that confinement begins to affect the equilibrium configuration of the molecule when the channel width $(H)$ is on the order of the free-solution radius of gyration $\left(S_{\text {bulk }}\right)$. Significant changes in the equilibrium relaxation time and diffusivity are observed at much larger widths.

\section{DNA MODEL}

In the present work, we extend our free-solution model of $\mathrm{DNA}^{21}$ for use in simulations of DNA in microfluidic devices. A linear molecule dissolved in a viscous solvent is represented by $N_{b}$ interaction sites (beads) connected through $N_{s}=N_{b}-1$ entropic connectors (springs). A force balance on this chain leads to the stochastic differential equation $^{19,21-23}$

$$
\begin{aligned}
& d \mathbf{r}=\left[\mathbf{u}+\frac{1}{k_{B} T} \mathbf{D} \cdot \mathbf{f}+\frac{\partial}{\partial \mathbf{r}} \cdot \mathbf{D}\right] d t+\sqrt{2} \mathbf{B} \cdot d \mathbf{w}, \\
& \mathbf{D}=\mathbf{B} \cdot \mathbf{B}^{T} .
\end{aligned}
$$

Here, $k_{B}$ is Boltzmann's constant, and $T$ is the absolute temperature. The vector $\mathbf{r}$ contains the $3 N_{b}$ spatial coordinates of the beads that constitute the polymer chain, with $\mathbf{r}_{i}$ denoting the three Cartesian coordinates of bead $i$. The vector $\mathbf{f}$ has length $3 N_{b}$, with $\mathbf{f}_{i}$ denoting the total non-Brownian, nonhydrodynamic force acting on bead $i$. The vector $\mathbf{u}$ of length $3 N_{b}$ represents the unperturbed velocity field (i.e., the velocity field in the absence of the polymer), with $\mathbf{u}_{i}=\mathbf{v}\left(\mathbf{r}_{i}\right)$ denoting the unperturbed velocity field at the location of bead $i$. The components of $d \mathbf{w}$ are obtained from a real-valued Gaussian distribution with mean zero and variance $d t$.

The motion of a segment of the macromolecule creates a velocity field in the fluid, which in turn affects the motion of the entire macromolecule. These hydrodynamic interactions (HI) enter the chain dynamics through the $3 \times 3$ block components of the $3 N_{b} \times 3 N_{b}$ diffusion tensor, $\mathbf{D}$, which can be expressed as

$$
\mathbf{D}_{i j}=k_{B} T\left(\frac{1}{6 \pi \eta a} \mathbf{I} \delta_{i j}+\boldsymbol{\Omega}_{i j}\right),
$$

where $\eta$ is the solvent viscosity, $a$ is the bead hydrodynamic radius, and $\boldsymbol{\Omega}_{i j}$ is the hydrodynamic interaction tensor, ${ }^{19,24}$ which relates the velocity perturbation at point $\mathbf{r}_{i}$ to a point force at $\mathbf{r}_{j}$. The Brownian forces,${ }^{25}$ which appear in the last term of Eq. (1), are intimately coupled to these velocity perturbations through the fluctuation-dissipation theorem [Eq. (2)].

In an infinite domain (no walls), the Stokes flow velocity field due to a point force located at $\mathbf{x}_{j}$ is given by $\mathbf{v}^{\prime}(\mathbf{x})$ $=\boldsymbol{\Omega}^{\mathrm{OB}}\left(\mathbf{x}-\mathbf{x}_{j}\right) \cdot \mathbf{f}\left(\mathbf{x}_{j}\right)$, where $\boldsymbol{\Omega}^{\mathrm{OB}}$ is the free-space Green's function, or Oseen-Burgers tensor, ${ }^{19,24}$

$$
\boldsymbol{\Omega}^{\mathrm{OB}}\left(\mathbf{x}-\mathbf{x}_{j}\right)=\frac{1}{8 \pi \eta\left|\mathbf{x}-\mathbf{x}_{j}\right|}\left[\mathbf{I}+\frac{\left(\mathbf{x}-\mathbf{x}_{i}\right)\left(\mathbf{x}-\mathbf{x}_{i}\right)}{\left|\mathbf{x}-\mathbf{x}_{j}\right|^{2}}\right] .
$$

The tensor, $\boldsymbol{\Omega}_{i j}$, appearing in Eq. (3) is then given by

$$
\boldsymbol{\Omega}_{i j}=\left(1-\delta_{i j}\right) \boldsymbol{\Omega}^{\mathrm{OB}}\left(\mathbf{r}_{i}-\mathbf{r}_{j}\right) .
$$

In Brownian dynamics simulations ${ }^{21,23,26}$ of polymers, $\boldsymbol{\Omega}^{\mathrm{OB}}$ is typically replaced with the Rotne-Prager-Yamakawa (RPY) tensor, ${ }^{27}$

$$
\begin{aligned}
& \mathbf{\Omega}^{\mathrm{RPY}}\left(\mathbf{x}-\mathbf{x}_{j}\right)=\frac{1}{8 \pi \eta\left|\mathbf{x}-\mathbf{x}_{j}\right|}\left[C_{1} \mathbf{I}+C_{2} \frac{\left(\mathbf{x}-\mathbf{x}_{i}\right)\left(\mathbf{x}-\mathbf{x}_{i}\right)}{\left|\mathbf{x}-\mathbf{x}_{j}\right|^{2}}\right] \\
& \text { if }\left|\mathbf{x}-\mathbf{x}_{j}\right| \geqslant 2 a, \\
& \frac{1}{6 \pi \eta a}\left[C_{1}^{\prime} \mathbf{I}+C_{2}^{\prime} \frac{\left(\mathbf{x}-\mathbf{x}_{i}\right)\left(\mathbf{x}-\mathbf{x}_{i}\right)}{\left|\mathbf{x}-\mathbf{x}_{j}\right|^{2}}\right] \text { if }\left|\mathbf{x}-\mathbf{x}_{j}\right|<2 a, \\
& C_{1}=1+\frac{2 a^{2}}{3\left|\mathbf{x}-\mathbf{x}_{j}\right|^{2}}, \quad C_{2}=1-\frac{2 a^{2}}{\left|\mathbf{x}-\mathbf{x}_{j}\right|^{2}}, \\
& C_{1}^{\prime}=1-\frac{9\left|\mathbf{x}-\mathbf{x}_{j}\right|}{32 a}, \quad C_{2}^{\prime}=\frac{3\left|\mathbf{x}-\mathbf{x}_{j}\right|}{32 a} .
\end{aligned}
$$

A diffusion tensor generated using RPY hydrodynamics is guaranteed to be positive-semidefinite for all chain configurations. We note here that, for both Oseen-Burgers and RPY hydrodynamics, $\boldsymbol{\Omega}_{i j}=0$ for $i=j$, and $\partial / \partial \mathbf{r}_{i} \cdot \boldsymbol{\Omega}_{i j}=0$ for all $i, j$. In Sec. III, we describe how the above discussion is modified for bounded domains.

The spring force between adjacent beads is described by means of a wormlike spring (WLS) model $^{28,29}$ which has been shown to be appropriate for molecules such as DNA having stiff backbones, ${ }^{21,26,29-34}$

$\mathbf{f}_{i j}^{s}=\frac{k_{B} T}{2 b_{k}}\left[\left(1-\frac{\left|\mathbf{r}_{j}-\mathbf{r}_{i}\right|}{q_{o}}\right)^{-2}-1+\frac{4\left|\mathbf{r}_{j}-\mathbf{r}_{i}\right|}{q_{o}}\right] \frac{\mathbf{r}_{j}-\mathbf{r}_{i}}{\left|\mathbf{r}_{j}-\mathbf{r}_{i}\right|}$.

Here, $\mathbf{f}_{i j}^{s}$ is the force exerted on bead $i$ due to connectivity with bead $j$, and $b_{k}$ is the Kuhn length of the molecule. Letting $N_{k}$ represent the number of Kuhn segments in the molecule and $N_{k, s}$ the number of Kuhn segments per spring, $q_{0}=N_{k, s} b_{k}$ is the maximum spring length, or the contour length of the portion of the molecule represented by one spring. The contour length of the chain is then given by $L$ $=N_{s} q_{0}$.

Following our previous work, ${ }^{21,26,30}$ for the excluded volume potential between two beads of the chain we use 


$$
U_{i j}^{\mathrm{ev}}=\frac{1}{2} v k_{B} T N_{k, s}^{2}\left(\frac{3}{4 \pi S_{s}^{2}}\right)^{3 / 2} \exp \left[\frac{-3\left|\mathbf{r}_{j}-\mathbf{r}_{i}\right|^{2}}{4 S_{s}^{2}}\right],
$$

where $v$ is the excluded volume parameter, and $S_{s}^{2}$ $=N_{k, s} b_{k}^{2} / 6$ is the radius of gyration of an ideal chain consisting of $N_{k, s}$ Kuhn segments.

Using $N_{s}=10$ for $21 \mu \mathrm{m}$ stained $\lambda$-phage DNA at room temperature in a $43.3 \mathrm{cP}$ solvent, in previous work we determined, ${ }^{21,30}$ by direct comparison to available bulk experimental data, that suitable parameter values are $b_{k}$ $=0.106 \mu \mathrm{m}, a=0.077 \mu \mathrm{m}, v=0.0012 \mu \mathrm{m}^{3}$. With these values, the above-outlined model was able to reproduce the experimentally observed bulk relaxation time, diffusivity and equilibrium stretch (size) of DNA. The model also gives results in quantitative agreement with transient and steadystate behavior of $21 \mu \mathrm{m}$ DNA in both simple shear and planar extension over a wide range of Weissenberg numbers. In contrast to other available treatments for DNA in solution, the model discussed here also produces diffusivity results in quantitative agreement with experimental data for chains ranging from 21 to $126 \mu \mathrm{m}$, underscoring its predictive capability. After appropriate modifications to account for confinement, the model should provide useful predictions of DNA behavior in microfluidic devices.

\section{HYDRODYNAMIC INTERACTIONS IN MICROFLUIDIC DEVICES}

As mentioned earlier, simple arguments predict that the dynamics of a dissolved macromolecule confined to a channel comparable to its equilibrium coil size $(\sim 1 \mu \mathrm{m}$ for viral DNA) are different from those in free solution, largely as a consequence of the no-slip boundary condition on the fluid motion. Available analytical work ${ }^{24,35,36}$ has concentrated on resolving particle-wall interactions for a few special geometries (i.e., a spherical particle near an infinite plane or located between two infinite planes, and a particle at the centerline of a cylindrical channel). The long-range effect of the wall on the mobility of a particle decays slowly, as $1 / h$, where $h$ is the distance from the wall. Hydrodynamic interactions between confined particles decay as $1 / r^{2}$ for particles near an infinite plane and exponentially in a channel. ${ }^{24,36}$ Dufresne $\mathrm{et} \mathrm{al}^{37}$ recently considered the hydrodynamic coupling of two Brownian particles near a plane wall. Those authors compared their experimental results to analytical predictions for point particles near an infinite plane, i.e., keeping only the leading order far field effects-and found good agreement with experimental observation provided $(h / a)$ $>2$, where $a$ is the radius of the particle. That study represents the first direct experimental validation of the use of point particle hydrodynamic interactions between Brownian particles near a surface. The findings of Dufresne et al. are particularly relevant to dynamical studies of polymers in confined geometries (microfluidic devices), where the velocity field generated by motion of the macromolecule is generally taken to be due to point forces acting on the interaction sites, or "beads" that constitute the chain.

We now present a method for the numerical evaluation of the hydrodynamic interaction tensor for general geom- etries. The level of treatment of $\mathrm{HI}$ in a bounded domain considered here is similar to that employed by Grier et al. ${ }^{37}$ in their work. Note however, that closed form solutions only exist for Oseen-Burgers hydrodynamics in a few special cases (i.e., point particles between infinite planes or in straight channels with constant cross-section); for general geometries the problem must be solved numerically.

The Green's function for Stokes flow in an arbitrary device can be expressed as

$$
\boldsymbol{\Omega}=\mathbf{\Omega}^{\mathrm{OB}}+\boldsymbol{\Omega}^{W},
$$

where $\boldsymbol{\Omega}^{\mathrm{OB}}$ is the free-space Green's function [Eq. (4)] and $\boldsymbol{\Omega}^{W}$ is a correction which accounts for the no-slip constraint on the walls. The velocity perturbation due to a point force acting at $\mathbf{x}_{j}$ is then given as

$$
\begin{aligned}
\mathbf{v}^{\prime}\left(\mathbf{x}, \mathbf{x}_{j}\right) & =\mathbf{v}_{\mathrm{OB}}^{\prime}\left(\mathbf{x}-\mathbf{x}_{j}\right)+\mathbf{v}_{W}^{\prime}\left(\mathbf{x}, \mathbf{x}_{j}\right) \\
& =\left[\boldsymbol{\Omega}^{\mathrm{OB}}\left(\mathbf{x}-\mathbf{x}_{j}\right)+\boldsymbol{\Omega}^{W}\left(\mathbf{x}, \mathbf{x}_{j}\right)\right] \cdot \mathbf{f}\left(\mathbf{x}_{j}\right) .
\end{aligned}
$$

Thus, $\mathbf{v}_{W}^{\prime}\left(\mathbf{x}, \mathbf{x}_{j}\right)$ can be obtained as the solution to the incompressible Stokes flow problem

$$
-\nabla p+\eta_{s} \nabla^{2} \mathbf{v}_{W}^{\prime}=0, \quad \nabla \cdot \mathbf{v}_{W}^{\prime}=0,
$$

subject to

$$
\mathbf{v}_{\mathrm{OB}}^{\prime}+\mathbf{v}_{W}^{\prime}=0 \text { at the walls. }
$$

The wall Green's function, $\boldsymbol{\Omega}^{W}\left(\mathbf{x}, \mathbf{x}_{j}\right)$, is obtained by the following procedure. First, we take $\mathbf{v}_{\mathrm{OB}}^{\prime}\left(\mathbf{x}-\mathbf{x}_{j}\right)$ to be due to a point force $\left(f_{1}\right)$ acting in the $x_{1}$ direction, located at the point $\mathbf{x}_{j}$. The Stokes flow problem is then solved using a finite element method ${ }^{49}$ to obtain $\mathbf{v}_{W}^{\prime}\left(\mathbf{x}, \mathbf{x}_{j}\right)$. This gives the first column of $\boldsymbol{\Omega}^{W}\left(\mathbf{x}, \mathbf{x}_{j}\right)$ by

$$
\left(\begin{array}{c}
\Omega_{11}^{W} \\
\Omega_{21}^{W} \\
\Omega_{31}^{W}
\end{array}\right)=\frac{1}{f_{1}} \mathbf{v}_{W}^{\prime} .
$$

Similarly, the second and third columns of $\boldsymbol{\Omega}^{W}\left(\mathbf{x}, \mathbf{x}_{j}\right)$ are obtained by applying point forces in the $x_{2}$ and $x_{3}$ directions, respectively. Now, $\boldsymbol{\Omega}_{i j}$, in Eq. (3) is given by

$$
\boldsymbol{\Omega}_{i j}=\boldsymbol{\Omega}^{W}\left(\mathbf{r}_{i}, \mathbf{r}_{j}\right)+\left(1-\delta_{i j}\right) \boldsymbol{\Omega}^{\mathrm{OB}}\left(\mathbf{r}_{i}-\mathbf{r}_{j}\right) .
$$

In contrast to the case of unbounded flow, here $\boldsymbol{\Omega}_{i j} \neq 0$ for $i=j$. Furthermore, $\partial / \partial \mathbf{r}_{i} \cdot \boldsymbol{\Omega}_{i j}^{W}$ is nonzero for $i=j$, resulting in a nonzero drift term in Eq. (1).

The evaluation of the Green's function is performed once, in a preprocessing step, for a given device or geometry; $\boldsymbol{\Omega}^{W}\left(\mathbf{x}_{i}, \mathbf{x}_{j}\right)$ is obtained numerically on a grid. During a Brownian dynamics simulation, $\boldsymbol{\Omega}_{i j}^{W}$ and its divergence are obtained by finite element interpolation. ${ }^{39}$

At first glance, it may appear that one needs $O\left(N_{G}^{2}\right)$ of the $\boldsymbol{\Omega}_{m n}^{W}$ 's, with $N_{G}$ being the number of grid points in the microfluidic domain. However, in confined geometries, the Green's function decays rapidly (exponentially, in a channel), and in practice one only needs to keep the $\boldsymbol{\Omega}_{m n}^{W}$ 's for $\left|\mathbf{x}_{n}-\mathbf{x}_{m}\right|<x_{0}$, where $x_{0}$ is a cutoff distance which depends on the proximity of the point $\mathbf{x}_{j}$ to the device walls. Thus, in practical applications, one needs $O\left(N_{G}\right)$ of the $\boldsymbol{\Omega}_{m n}^{W}$ 's, with the coefficient of proportionality depending on the details of the computational domain. 
As in the bulk case (Sec. II), use of the point force Green's function in the above-given formulation leads to nonpositive-semidefinite $\mathbf{D}$ for some chain configurations. However, a diffusion tensor constructed by the aboveoutlined method using $\boldsymbol{\Omega}^{\mathrm{RPY}}$ in place of $\boldsymbol{\Omega}^{\mathrm{OB}}$ results in a nonsymmetric $\mathbf{D}$; this violates the reciprocity relation

$$
\boldsymbol{\Omega}\left(\mathbf{r}_{i}, \mathbf{r}_{j}\right)=\boldsymbol{\Omega}^{T}\left(\mathbf{r}_{j}, \mathbf{r}_{i}\right),
$$

which follows from self-adjointness of the Stokes operator. ${ }^{24}$ This violation is analogous to that which would occur if RPY hydrodynamic interactions were used naively for spheres of unequal size ${ }^{40-42}$ For that situation, Felderhof ${ }^{40}$ performed a multipole expansion of the free-solution hydrodynamic interaction tensor. To order $1 / r^{3}$, the resulting hydrodynamic interaction tensor is identical (for $\left|r_{i j}\right| \geqslant 2 a$ ) to the symmetrized RPY tensor, $\boldsymbol{\Omega}_{i j}^{\mathrm{MP}}=\left(\boldsymbol{\Omega}_{i j}^{\mathrm{RPY}}+\boldsymbol{\Omega}_{j i}^{\mathrm{RPY}}\right) / 2$. By analogy with this result, we obtain a symmetric, positive-semidefinite diffusion tensor as follows. First, $\boldsymbol{\Omega}_{i j}^{\mathrm{OB}}$ is replaced by $\boldsymbol{\Omega}_{i j}^{\mathrm{RPY}}$ in Eqs. (15) and (17), giving a wall correction $\overline{\boldsymbol{\Omega}}_{i j}^{W} \neq\left(\overline{\mathbf{\Omega}}_{j i}^{W}\right)^{T}$. Then, a symmetric, positive-semidefinite diffusion tensor is calculated according to

$$
\boldsymbol{\Omega}_{i j}^{W}=\frac{\overline{\boldsymbol{\Omega}}_{i j}^{W}+\left(\overline{\mathbf{\Omega}}_{j i}^{W}\right)^{T}}{2} .
$$

We note here that we account for hydrodynamic interactions at the point force level (i.e., Oseen-Burgers in a confined geometry); the Rotne-Prager-Yamakawa formulation is employed only to maintain positive-semidefiniteness of the diffusion tensor. While the use of Eq. (19) to obtain a symmetric $\mathbf{D}$ is not rigorous, it is essentially a near-field regularization, and introduces an error in the point force hydrodynamics of order $a / H \ll 1$. Our treatment of hydrodynamic interaction may be considered as an approximate Rotne-Prager-Yamakawa formulation for confined geometries. For the special case of particles above an infinite plane wall, our treatment of the hydrodynamic interaction can be worked out analytically. We have done this and verified that our method is indeed a well-behaved near-field modification of the near-wall point-force solution.

\section{SIMULATION}

In this work, we consider the behavior of individual DNA molecules in an infinitely long microchannel with square cross section (see Fig. 1). The centerline of the channel is oriented along the $x$ axis, with the cross section lying in the $y z$ plane. In the limit of infinite channel length, a point force acting on the fluid generates no net flow even if the channel ends are open; for there to be a net flow the force would need to be infinite. For the numerical evaluation of the hydrodynamic interaction tensor, the infinitely long channel is therefore approximated by a closed channel of length $10 \mathrm{H}$, at which the results are insensitive to further increase in length. Figure 2 shows the mesh and $v_{x}=0.01 / \zeta$ (red) and $-0.01 / \zeta$ (blue) contour surfaces for a unit point force in the $x$ direction, located at the center of a 6.3- $\mu \mathrm{m}$-wide channel. In addition to the detailed "HI" model described earlier, we consider two approximate models. In the reduced mobility (RM) model, hydrodynamic interactions between particles

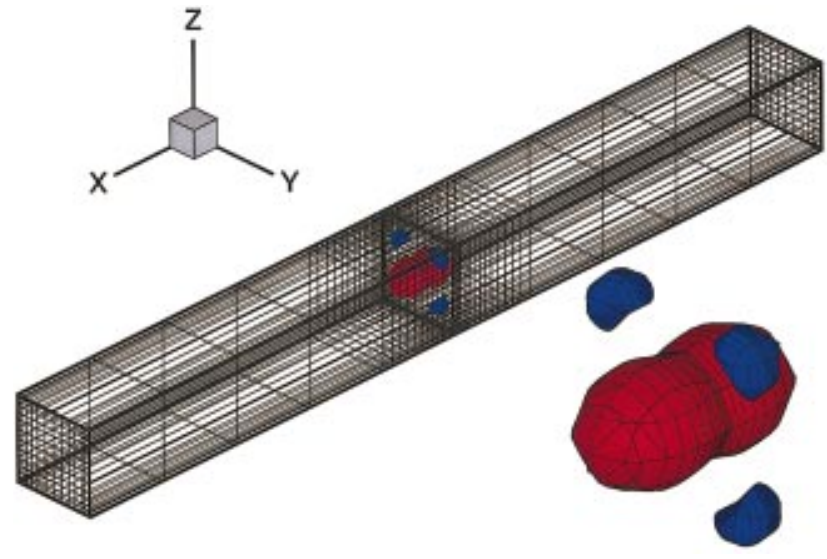

FIG. 2. (Color) Green's function meshing for a $63.6 \mu \mathrm{m} \times 6.36 \mu \mathrm{m} \times 6.36$ $\mu \mathrm{m}$ channel. Also shown is the $v_{x}=0.01 / \zeta$ (red) and $-0.01 / \zeta$ (blue) contours due to a point force in the $x$ direction of magnitude 1 , located at the center of the channel. The inset is a closeup view of the velocity contours.

are neglected, while modifications to the bead mobility due to the wall are maintained. In that case the diffusion tensor [Eq. (3)] becomes block-diagonal,

$$
\mathbf{D}_{i j}^{\mathrm{RM}}=k_{B} T \delta_{i j}\left(\frac{1}{6 \pi \eta a} \mathbf{I}+\mathbf{\Omega}_{i j}^{W}\right) .
$$

In the free-draining (FD) model, which has been favored in many literature studies of DNA, both hydrodynamic interactions and modifications to the bead mobility are neglected, resulting in a position-independent, isotropic diffusion tensor,

$$
\mathbf{D}_{i j}^{\mathrm{FD}}=\delta_{i j} \frac{k_{B} T}{6 \pi \eta a} \mathbf{I} .
$$

The physical confinement of the molecule is taken into account through a simple bead-wall repulsive potential of the form

$$
U_{i}^{\text {wall }}=\left\{\begin{array}{l}
\frac{A_{\text {wall }}}{3 b_{k} \delta_{\text {wall }}^{2}}\left(y-\delta_{\text {wall }}\right)^{3} \quad \text { for } y<\delta_{\text {wall }}, \\
0 \quad \text { for } y \geqslant \delta_{\text {wall }},
\end{array}\right.
$$

where $y$ represents the distance of bead $i$ from the wall in the wall-normal direction (into the fluid). Throughout this work, we take $A_{\text {wall }}=25 k_{B} T$ and $\delta_{\text {wall }}=b_{k} N_{k, s}^{1 / 2} / 2=0.236 \mu \mathrm{m}$.

Equation (1) was evolved in time using a semi-implicit integration scheme described in our previous papers..$^{21,23}$ The range of the hydrodynamic interaction is proportional to the channel width, $H$; all components of $\mathbf{D}$ less than $0.001 \max \left(D_{i i}\right)$ were neglected. The decomposition of $\mathbf{D}$ [Eq. (3)] is computationally demanding, and was achieved using a fast algorithm proposed by Fixman ${ }^{25}$ and implemented as described in our earlier work. ${ }^{23}$ A time step of $\Delta t=0.01\left(6 \pi \eta a S_{s}^{2}\right) /\left(k_{B} T\right)$ was used in all simulations. All simulations here were performed without an imposed velocity field $(\mathbf{v}=0)$. All results are presented for DNA at room temperature in a $1 \mathrm{cP}$ solvent. Chains ranging from $4.2 \mu \mathrm{m}$ $\left(N_{s}=2\right)$ to $420 \mu \mathrm{m}\left(N_{s}=200\right)$ were simulated in channels 


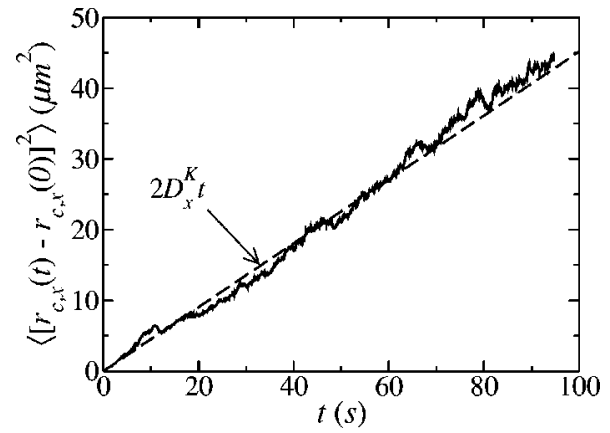

FIG. 3. Comparison between the mean-squared displacement of the chain center of mass (solid line) in the axial direction and that predicted by the Kirkwood diffusivity (dashed line). Results are for a $21 \mu \mathrm{m}$ chain in a $1.59-\mu \mathrm{m}$-wide channel. The mean-squared displacement was averaged over 80 trajectories.

having widths in the range of $0.636-21.2 \mu \mathrm{m}$. Note that the level of molecular discretization was kept constant at $N_{k, s}$ $=19.8$. We investigated the effect of molecular discretization on the full model by using $N_{k, s}=13.2$ in simulations of 42 , 126 , and $210 \mu \mathrm{m}$ chains in $1.6 \mu \mathrm{m}$ channels; the results were within a few percent of the coarser representation, lending credence to the predictive capabilities of the model. The results were also found to be fairly insensitive to the wall parameter $\delta$, provided $\delta=O(a)$.

The following properties are considered in this work. The "stretch" of the chain is defined as the absolute length of the molecule in the channel direction

$$
\bar{X}=\max \left(r_{i, x}\right)-\min \left(r_{i, x}\right),
$$

where $r_{i, x}$ is the $x$ component of the position vector of bead $i ; X=\langle\bar{X}\rangle$ The equilibrium diffusivity of a chain in the channel direction, $D_{x}$, is determined by

$$
D_{x}=\lim _{t \rightarrow \infty} \frac{1}{2 t}\left\langle\left[r_{c, x}(t)-r_{c, x}(0)\right]^{2}\right\rangle,
$$

where $r_{c, x}$ is the $x$ component of the center-of-mass of the chain. The approximate Kirkwood diffusivity tensor is given by

$$
\mathbf{D}^{K}=\left\langle\frac{1}{N_{b}^{2}} \sum_{i, j=1}^{N_{b}} \mathbf{D}_{i j}\right\rangle .
$$

The Kirkwood diffusivity in the channel direction is $D_{x}^{K}$ $=D_{11}^{K}$. We have compared the Kirkwood diffusivity to the center-of-mass diffusivity calculated by Eq. (25), and found them to be in quantitative agreement for all channels considered in this work. Figure 3 shows a comparison between the Kirkwood diffusivity and that obtained by Eq. (25) for a 21 $\mu \mathrm{m}$ DNA chain in a $1.59-\mu \mathrm{m}$-wide channel.

The longest relaxation time of a chain, $\lambda_{1}$, is calculated by allowing a chain that is initially fully stretched along the centerline of the channel to relax to equilibrium. Near equilibrium, the relaxation time is determined by a fit to an exponential decay,

$$
\left\langle\bar{X}^{2}\right\rangle(t)=\left(\bar{X}^{2}(0)-\left\langle\bar{X}^{2}\right\rangle_{\mathrm{eq}}\right) \exp \left(-\frac{t}{\lambda_{1}}\right)+\left\langle\bar{X}^{2}\right\rangle_{\mathrm{eq}},
$$

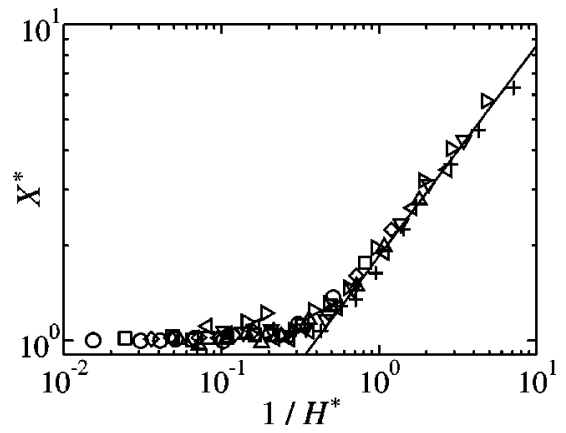

FIG. 4. Stretch as a function of channel width for various lengths of DNA. Symbols correspond to $L=4.2 \mu \mathrm{m}(\bigcirc), L=10.5 \mu \mathrm{m}(\square), L=21 \mu \mathrm{m}(\diamond)$, $L=42 \mu \mathrm{m}(\triangle), L=84 \mu \mathrm{m}(\triangleleft), L=126 \mu \mathrm{m}(\nabla), L=210 \mu \mathrm{m}(\triangleright)$, and $L$ $=420 \mu \mathrm{m}(+)$. The solid line corresponds to the scaling $X^{*} \propto\left(1 / H^{*}\right)^{2 / 3}$.

where \langle\rangle$_{\text {eq }}$ indicates an equilibrium average. The radius of gyration of the chain, $S$, is defined through

$$
S^{2}=\left\langle\frac{1}{N_{b}} \sum_{i=1}^{N_{b}}\left|\mathbf{r}_{i}-\mathbf{r}_{c}\right|^{2}\right\rangle \text {. }
$$

We also define $X_{b}, D_{x, b}^{\mathrm{K}}, \lambda_{1, b}$, and $S_{b}$ as the equilibrium bulk values of the stretch, Kirkwood diffusivity in the channel direction, longest relaxation time, and radius of gyration, respectively.

\section{RESULTS}

We first present results on the equilibrium stretch of DNA molecules in microchannels. Simple scaling arguments $^{8}$ give $X \propto N_{k} H^{-2 / 3}$ for a chain in a good solvent; this scaling has been previously verified by Monte Carlo simulations. ${ }^{8,9}$ Figure 4 shows the dimensionless equilibrium stretch, $X^{*}=\langle X\rangle_{\mathrm{eq}} / X_{b}$, as a function of the inverse dimensionless channel width, $1 / H^{*}=S_{b} / H$. Chains ranging from $4.2 \mu \mathrm{m}\left(N_{s}=1\right)$ to $420 \mu \mathrm{m}\left(N_{s}=200\right)$ are considered. Also shown is the predicted scaling, $X^{*} \propto\left(H^{*}\right)^{-2 / 3}$. As found previously, ${ }^{9}$ static confinement effects appear here at $1 / H^{*}$ $\approx 0.3$, while the scaling regime is realized for $1 / H^{*} \geq 0.5$. The transition from free-solution to confined behavior is centered about the point $1 / H^{*} \approx 0.4$. For $1 / H^{*} \gtrsim 0.4$, we refer to the chains as strongly confined, while below the transition we refer to the chains as weakly confined.

Although static equilibrium properties such as the stretch can be obtained through Monte Carlo simulations, dynamic properties such as relaxation time and diffusivity require the resolution of solvent motion, as do transient processes like the dynamics of a relaxing chain. Figure 5 shows the dimensionless Kirkwood diffusivity, $D^{*}=D_{x}^{K} / D_{x, b}^{K}$, as a function of $1 / H^{*}$ for chains ranging from 4.2 to $420 \mu \mathrm{m}$. For $a$ $\ll H, a \ll S_{b}$, and $N \gg 1$, dimensional analysis leads one to expect $D^{*}=f\left(H^{*}\right)$. This will be discussed in more detail in the following. The results shown in Fig. 5 were obtained by FD simulation, with the diffusion tensor and corresponding Kirkwood diffusivity periodically evaluated from an instantaneous realization of the static equilibrium configuration.

Figure 5 shows a broad crossover from free-solution behavior. In the weakly confined region $\left(1 / H^{*} \leq 0.4\right)$, the molecule might be expected to behave as a sphere diffusing 


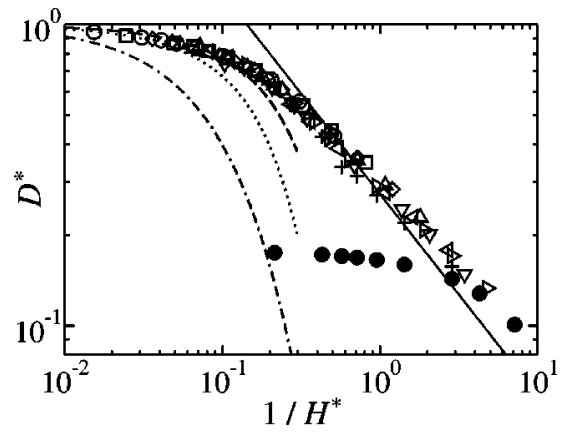

FIG. 5. Kirkwood diffusivity as a function of inverse channel width for various lengths of DNA. Symbols correspond to $L=4.2 \mu \mathrm{m}(\bigcirc), L=10.5$ $\mu \mathrm{m}(\square), L=21 \mu \mathrm{m}(\diamond), L=42 \mu \mathrm{m}(\triangle), L=84 \mu \mathrm{m}(\triangleleft), L=126 \mu \mathrm{m}(\nabla)$, $L=210 \mu \mathrm{m}(\triangleright)$, and $L=420 \mu \mathrm{m}(+)$. Line styles correspond to $D^{*}$ $\propto\left(1 / H^{*}\right)^{-2 / 3}$ (solid line), Faxen's expression (dashed line), the curve fit of the data of Cannell and Rondelez (Ref. 45) (dotted line) and the curve fit of the data of Satterfield et al. (Ref. 44) (dot-dashed). The closed symbols are the RM model results for the $420 \mu \mathrm{m}$ chains.

in a large channel. The special case of a sphere diffusing along the centerline of a circular channel has been solved to varying degrees of accuracy. ${ }^{35}$ The asymptotic solution of Faxen $^{43}$ is given by

$D^{*}=1-2.104\left(\frac{r_{s}}{r_{p}}\right)+2.09\left(\frac{r_{s}}{r_{p}}\right)^{3}-0.95\left(\frac{r_{s}}{r_{p}}\right)^{5}+O\left(\frac{r_{s}}{r_{p}}\right)^{7}$,

where $r_{s}$ and $r_{p}$ are the sphere and channel radii, respectively. From our bulk simulations, we determined the hydrodynamic radius $\left(r_{h}\right)$ of our chains to be $0.55 S_{b}$. The dashed curve in Fig. 5 represents Eq. (29) with $r_{s}=r_{h}$ and $r_{p}$ $=H / 2$. We find that Faxen's solution matches well our results for chain molecules when $1 / H^{*}<0.1$. In comparison, for solid spheres in a circular channel, Faxen's expression is relatively accurate out to $r_{s} / r_{p}=1 / 2$, which would correspond to $1 / H^{*} \approx 0.45$. Our results lie slightly below Faxen's expression for small $1 / H^{*}$, probably because we do not force the chains to remain centered in the channel. The good agreement with Faxen's expression indicates that in the region $1 / H^{*}<0.1$ the molecule behaves as a nondraining chain with the decrease in mobility due to channel walls affecting the entire chain uniformly. In the region $0.1 \leqq 1 / H^{*} \leq 0.4$, the chain can no longer be represented as sphere diffusing in a channel, and the mobility becomes higher than predicted by the Faxen result; hydrodynamic screening due to channel walls causes a transition toward free-draining behavior. This transition from nondraining to free-draining behavior begins at $H \approx 10 S_{b}$, well before the transition to strongly confined behavior $\left(H \approx 2 S_{b}\right)$ in Fig. 4.

Before discussing our results for larger $1 / H^{*}$, it is of interest to review several experimental investigations in the region $1 / H^{*}<0.5$. All available experiments on porous materials measure an effective diffusivity $\left(D_{\text {eff }}\right)$. This quantity is related to our reduced diffusivity by ${ }^{44} D_{\text {eff }}$ $=D_{b} \theta K_{p} D^{*} / \tau$, where $D_{b}$ is the bulk diffusivity, $\theta$ is the volume fraction of the pores, $\tau$ is the tortuosity, and $K_{p}$ is the equilibrium partition coefficient. In 1973, Satterfield et al. ${ }^{44}$ reported on the diffusion of low molecular weight paraffins, aromatic hydrocarbons, and sugars through homogeneous microporous silica-alumina bead catalyst. For $r_{s} / r_{p}<1 / 2$, they found their data could be fit to the expression $D^{*}$ $=\exp \left(-4.6 r_{s} / r_{p}\right)$. This relation is shown as the dot-dashed curve in Fig. 5. In that work they noted that this exponential dependence on $r_{s} / r_{p}$ had also been observed in other systems. In 1975, Colton et al. ${ }^{38}$ reported on the diffusion of proteins and monodisperse polystyrene (molecular weights up to 670000$)$ through borosilicate glass. They found that $D^{*}$ for the proteins was in qualitative agreement with the above-given exponential fit, but that $D^{*}$ for the polystyrene chains was independent of $H^{*}$. In 1980, Cannell and Rondelez $^{45}$ considered the diffusion of monodisperse polystyrene chains (molecular weights up to 600000 ) through porous membranes. For $0.1<r_{s} / r_{p}<0.5$, they found their data followed the Renkin equation ${ }^{46}$

$$
D^{*} K_{p}=\left(1-\frac{r_{s}}{r_{p}}\right)^{2}\left[1-2.104 \frac{r_{s}}{r_{p}}+2.09\left(\frac{r_{s}}{r_{p}}\right)^{3}-0.95\left(\frac{r_{s}}{r_{p}}\right)^{5}\right] \text {, }
$$

provided they set $r_{s}=1.45 r_{h}$, where $r_{h}$ was the StokesEinstein hydrodynamic radius of the molecules as determined from light-scattering experiments. The Renkin equation combines Ferry's equilibrium partition coefficient, ${ }^{44,47}$ $K_{p}^{F}=\left(1-r_{s} / r_{p}\right)^{2}$, with Faxen's expression for the diffusion of a sphere along the centerline of a channel. The dotted curve in Fig. 5 represents Faxen's expression with $r_{s}$ $=1.45 r_{h}$. The Renkin equation has been used with varying success to describe rigid solute diffusion through biological and artificial membranes (see Satterfield et al. ${ }^{44}$ for references).

We note that the curve representing the experimental study of the diffusion of long flexible polymers through membranes lies below our simulation results (the other study $^{38}$ concluded no dependence on $H^{*}$ ). Our simulations provide a direct measurement of $D^{*}$, unlike the experimental studies which inferred $D^{*}$ from indirect estimates of $\tau, \theta$, and $K_{p}$. In addition, our simulations are performed at infinite dilution, while the experimental investigations, although performed at finite concentrations, assumed $D^{*}$ (and $K_{p}$ ) to be independent of solute concentration within the pores. This is in contrast to the study of Cannell and Rondelez, ${ }^{44}$ who observed a large change in $K_{p} D^{*}$ when the (dilute) concentration was increased by a factor of 3 . Also, in our simulations, there is no adsorption of the solute to the pore walls. In the experiments of Colton and Satterfield, preferential solute adsorption was estimated from equilibrium studies, and the estimated corrections to the pore diameters were made when necessary. Cannell and Rondelez measured the flow rate of polystyrene solutions through their membranes as a function of time and concluded that adsorption did not occur. Furthermore, all of the above-discussed experiments were obtained from membranes having pore radii on the order of nanometers; the diffusivity determined from those experiments may include effects of potential wells. 
We now turn our discussion to the "strongly confined" region of Fig. $5\left(1 / H^{*}>0.5\right)$. In 1977 , Brochard and de Gennes ${ }^{16}$ used a modified blob theory to obtain the scaling relation $D^{*} \propto\left(1 / H^{*}\right)^{-2 / 3}$, provided that $a \ll H \ll S_{b}$. In developing this relation, they assumed that the highly confined molecule could be represented as a sequence of blobs which do not interact hydrodynamically. This assumption imposes the scaling $D \propto N_{k}^{-1}$. In fact, if $a \ll H, D \propto N_{k}^{-1}$ and $D^{*} \propto\left(H^{*}\right)^{m}$, then dimensional analysis and good solvent bulk molecular weight scaling are sufficient to arrive at $m$ $=2 / 3$.

More recently, Harden and Doi ${ }^{17}$ combined an analytical-series solution for the Stokes flow due to point forces in a capillary (circular cross section) with a selfconsistent mean field calculation for the equilibrium configuration of the confined chain. They obtained $D \propto N_{k}^{-1}$ for highly confined chains, but found that transverse averaging of the Kirkwood diffusion coefficient results in $D^{*}$ $\propto\left(H^{*}\right)^{0.61}$. Note that the results of Harden and Doi indicate that the reduced variable $D^{*}$ will not display a master curve in $1 / H^{*}$ for highly confined chains. But as mentioned earlier, if the scale $a$ does not enter the problem, the exponent must be $2 / 3$. However, in the work of Harden and Doi and in our work presented here, there is an additional ratio of length scales $a / \delta$ that comes into play, where $\delta$ is the length scale associated with polymer-wall interactions. The ratio $a / \delta$ is order unity, which suggests that the $2 / 3$ scaling proposed by Brochard and de Gennes cannot be realized; the magnitude of the exponent will depend on the magnitude of $a / \delta$. The fact that Harden and Doi's exponent of 0.61 persists for $H / a>10^{2}$ is consistent with this reasoning, as are our results, which we now discuss.

Our results collapse onto a master curve in $D^{*}$ versus $H^{*}$ in the weakly confined region. This master curve extends beyond the region represented by Faxen's expression, all the way to the transition to strongly confined behavior. However, in the strongly confined region $\left(1 / H^{*} \gtrsim 0.4\right)$, we observe some scatter in the data ( $D^{*}$ lower for longer chains) as well as a deviation from the predicted scaling for the diffusivity data; we find $D^{*} \sim\left(1 / H^{*}\right)^{-1 / 2}$ from a fit of the data in this region, rather than $D^{*} \sim\left(1 / H^{*}\right)^{-2 / 3}$. As discussed earlier, this deviation indicates that additional length scales (i.e., the ratio $a / \delta$ ) are coming into play that were not accounted for in the simple scaling theory of Brochard and de Gennes.

As the level of chain confinement increases, hydrodynamic interactions between beads become weaker due to the solvent boundary condition on the walls of the device. To quantify this effect, we performed simulations using the RM model [Eq. (20)], which assumes complete screening of interbead hydrodynamic interactions. The diffusivity of 420 $\mu \mathrm{m}$ DNA chains using the RM model are shown as closed circles in Fig. 5. The RM model results converge to those of the full model at $S_{b} \geq 5 \mathrm{H}$. In general, the point at which the RM results match those of the full model is a function of the level of molecular discretization $\left(N_{k, s}=19.8\right.$, for the results reported in this work). When $H$ is on the order of the equilibrium spring length $\left(\approx b_{k} N_{k, s}^{1 / 2}\right)$, hydrodynamic interactions between beads are screened. However, at that level of confinement, a finer molecular model (smaller $N_{k, s}$ ) should be
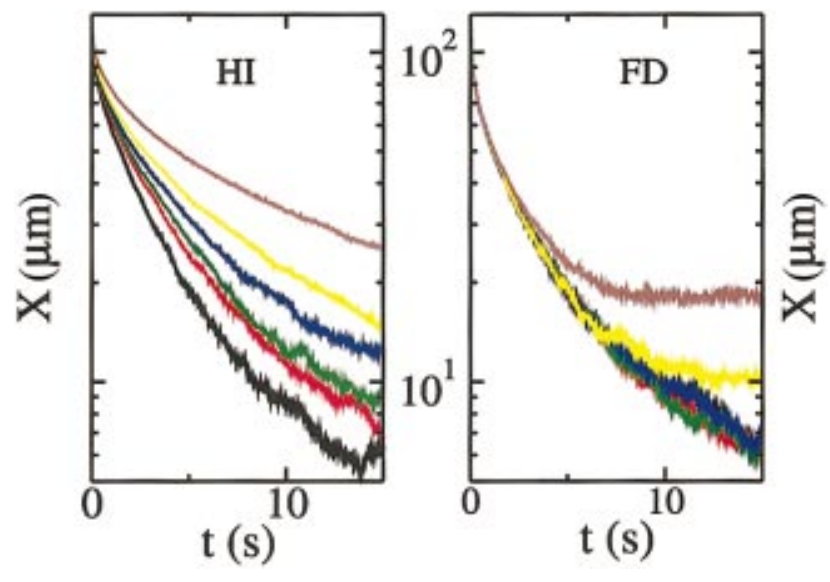

FIG. 6. (Color) Relaxation of $126 \mu \mathrm{m}$ chains, initially $99 \%$ stretched along the centerline of the channel. Results for both the HI and FD models are shown. The time axis for the FD case has been scaled so that the two models have equivalent bulk relaxation times (see the text). Each data set represents an average over 10 trajectories. Line colors correspond to channel widths of $H \rightarrow \infty \quad$ (black), $\quad H=10.6 \mu \mathrm{m}=4.8 S_{\text {bulk }} \quad$ (red), $H=6.36 \mu \mathrm{m}=2.9 S_{\text {bulk }}$ (green), $H=3.18 \mu \mathrm{m}=1.5 S_{\text {bulk }}$ (blue), $H=1.59 \mu \mathrm{m}=0.73 S_{\text {bulk }}$ (orange), $H=0.636 \mu \mathrm{m}=0.29 S_{\text {bulk }}$ (brown).

used, at which point screening will again no longer be complete. The RM model is thus of limited value.

We next consider the relaxation of DNA chains in microchannels. Individual chains, initially $99 \%$ stretched along the axis of the channel, were allowed to relax to equilibrium. Figure 6 shows the transient stretch of $126 \mu \mathrm{m}$ DNA chains, averaged over 10 trajectories, for various channel widths. Both the HI and FD model results are shown. Recall that model parameters were chosen so that relaxation times of both the HI and FD models matched the experimental bulk relaxation time for $21 \mu \mathrm{m}$ DNA chains. In order to provide a more reasonable comparison between the two models, the time axis of the FD results was scaled by $\lambda_{\mathrm{HI}, b}^{126} / \lambda_{\mathrm{FD}, b}^{126} \approx 0.6$, where $\lambda_{\mathrm{HI}, b}^{126}$ and $\lambda_{\mathrm{FD}, b}^{126}$ are the bulk relaxation time of $126 \mu \mathrm{m}$ chains as determined by the HI and FD models, respectively. The HI relaxation dynamics are strongly dependent on channel width, while the relaxation dynamics of the FD model are virtually unaffected by confinement. The RM model (results not shown) overpredicts the relaxation times; the HI and RM relaxation results converge at the same point as the diffusivity results in Fig. 5.

Longest chain relaxation times were determined by the method leading to Eq. (27) for various chain lengths and channel widths. Figure 7 shows the reduced relaxation time, $\lambda_{1}^{*}=\lambda_{1} / \lambda_{1, b}$ as a function of $1 / H^{*}$ for chains up to $126 \mu \mathrm{m}$. We observe a crossover region centered about $1 / H^{*} \approx 0.1$ followed by a power law region which is fully developed at $1 / H^{*} \approx 0.5$. We note two sources of scatter in the $\lambda_{1}$ data obtained in the crossover region. First, chains in this region may be fully relaxed before they have had time to sample the cross section of the channel; the relaxation time obtained by Eq. (27) in this region is not a true equilibrium average (such as that obtained from the autocorrelation function for the end-to-end vector). Second, there is significantly more noise in the relaxation trajectories in this region.

The only existing theoretical prediction for the longest 


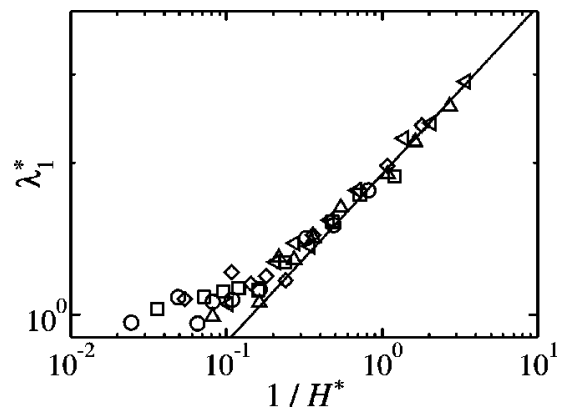

FIG. 7. Chain relaxation time as a function of channel width for various lengths of DNA. Symbols correspond to $L=10.5 \mu \mathrm{m}(\bigcirc), L=21 \mu \mathrm{m}(\square)$, $L=42 \mu \mathrm{m}(\diamond), L=84 \mu \mathrm{m}(\triangle), L=126 \mu \mathrm{m}(\triangleleft)$. The solid line corresponds to the scaling $\lambda^{*} \propto\left(1 / H^{*}\right)^{1 / 3}$.

relaxation time is for highly confined chains, and again comes from the scaling analysis of Brochard and de Gennes. ${ }^{16}$ By assuming complete screening of blob-blob hydrodynamic interactions, they predicted $\lambda_{1} \propto N_{k}^{2} H^{-1 / 3}$. As in the case of diffusivity, dimensional analysis for the case of weak confinement leads to a master curve in the reduced variables $\lambda_{1}^{*}$ and $1 / H^{*}$. If no new length scales come into play in the highly confined region, we again expect a master curve in the reduced variables. Since Rouse-type scaling $\left(\lambda_{1} \propto N_{k}^{2}\right)$ is also expected in this region, one obtains (using bulk good solvent scaling, $\lambda_{1, b} \propto N_{k}^{9 / 5}$ and $S_{b} \propto N_{k}^{3 / 5}$ ) a power law in the reduced variables $\lambda_{1}^{*} \propto\left(1 / H^{*}\right)^{1 / 3}$. This prediction is shown as the solid line in Fig. 7, which is in good agreement with our results. As anticipated by Brochard and de Gennes, ${ }^{16}$ for strongly confined chains the relaxation time shows a weaker dependence on channel width than does the stretch or diffusivity.

The relaxation of a stretched chain in a narrow slit has been observed experimentally by Bakajin et al. ${ }^{48}$ In that work, fluorescently dyed DNA molecules were allowed to relax to equilibrium after being stretched in an electric field. Those authors found that confinement significantly increased the time required for individual molecules to relax to equilibrium. ${ }^{49}$ For $\approx 74 \mu \mathrm{m}$ chains, Bakajin et al. estimated that the relaxation time roughly doubled when the slit width was decreased from 5 to $0.3 \mu \mathrm{m}$, and again when the slit width was decreased to $0.09 \mu \mathrm{m}$. We estimate $S_{b}=1.6 \mu \mathrm{m}$ for $74 \mu \mathrm{m}$ chains, which gives $1 / H^{*}=0.32,5.3$, and 17.8 for the 5, 0.3, and $0.09 \mu \mathrm{m}$ slits, respectively. Figure 7 indicates that $\lambda^{*}$ does increase by about a factor of 2 in going from $1 / H^{*}=0.32$ to $1 / H^{*}=5.3$ in agreement with the observations of Bakajin et al. From the power law scaling (for which the exponents for slit and channel are expected to be the same), one expects that $\lambda *$ will increase by a factor of 1.5 as $1 / H^{*}$ increases from 5.3 to 17.8 .

\section{CONCLUSIONS}

We have performed self-consistent Brownian dynamicshydrodynamic simulations of DNA in microchannels, using a method that simultaneously resolves both macromolecular and solvent motion in microfluidic devices. For diffusivity in the axial direction $\left(D_{x}^{K}\right)$ and longest relaxation time $\left(\lambda_{1}\right)$, we observe a crossover region from free-solution dynamics cen- tered about the point $H \approx 10 S_{b}$, where $H$ is the width of the square channel and $S_{b}$ is the free-solution radius of gyration of the chain. For $H \geqslant 10 S_{b}$, we find that the molecule diffuses in a manner similar to a solid sphere with radius equal to the free-solution hydrodynamic radius of the molecule. At $H \approx 10 S_{b}$ the molecule begins a transition to free-draining behavior, which is complete at $H \approx 2 S_{b}$. For $H \leqslant 2 S_{b}$, the chains may be considered strongly confined and Rouse-type. In the strongly confined region we find the equilibrium length $(X)$ and $\lambda_{1}$ to be well-represented by the scaling relations $X \propto N_{k} H^{2 / 3}$ and $\lambda_{1} \propto N_{k}^{-2} H^{-1 / 3}$ as predicted by Brochard and de Gennes. ${ }^{16}$ The computed diffusivity in the strongly confined region does not follow the predicted scaling of Brochard and de Gennes, ${ }^{16} D_{x}^{K} \propto N_{k}^{-1} H^{-2 / 3}$. While we do observe Rouse-type behavior $\left(D_{x}^{K} \propto N_{k}^{-1}\right)$, we find $D \propto H^{-1 / 2}$. Our diffusion results, as well as those of Harden and Doi, ${ }^{17}$ suggest that the diffusivity scaling exponent also depends on the ratio $a / \delta$, where $a$ is the segment hydrodynamic radius and $\delta$ is the length scale associated with polymer-wall interactions.

\section{ACKNOWLEDGMENTS}

We gratefully acknowledge the support of the NSF Nanoscale Modeling and Simulation Program, and the Department of Energy.

${ }^{1}$ O. S. Andersen, Biophys. J. 77, 2899 (1999).

${ }^{2}$ C. F. Chou, R. H. Austin, O. Bakajin et al., Electrophoresis 21, 81 (2000).

${ }^{3}$ A. Lim, E. T. Dimalanta, K. D. Potamousis et al., Genome Res. 11, 1584 (2001).

${ }^{4}$ N. Perna, G. Plunkett III, V. Burland et al., Nature (London) 409, 529 (2001).

${ }^{5}$ M. Sauer, B. Angerer, W. Ankenbauer, Z. Földes-Papp, F. Göbel, K. T. Han, R. Rigler, A. Schultz, J. Wolfrum, and C. Zander, J. Biotechnol. 86, 181 (2001).

${ }^{6}$ M. Hinz, S. Gura, B. Nitzan, S. Margel, and H. Seliger, J. Biotechnol. 86, 281 (2001).

${ }^{7}$ J. Stephan, K. Dörre, S. Brakmann, T. Winkler et al., J. Biotechnol. 86, 255 (2001).

${ }^{8}$ F. T. Wall, W. A. Seitz, J. C. Chin, and P. G. de Gennes, Proc. Natl. Acad. Sci. U.S.A. 75, 2069 (1978).

${ }^{9}$ J. H. van Vliet and G. ten Brinke, J. Chem. Phys. 93, 1436 (1990).

${ }^{10}$ D. C. Schwartz and C. R. Cantor, Cell 37, 67 (1984).

${ }^{11}$ D. C. Schwartz and M. Koval, Nature (London) 338, 520 (1989).

${ }^{12}$ J. L. Viovy, Rev. Mod. Phys. 72, 813 (2000).

${ }^{13}$ R. M. Kantor, X. H. Guo, E. J. Huff, and D. C. Schwartz, Biochem. Biophys. Res. Commun. 258, 102 (1999).

${ }^{14}$ P. J. Shrewsbury, D. Liepmann, and S. J. Muller, Biomed. Microdevices 4, 17 (2002).

${ }^{15}$ P. J. Shrewsbury, S. J. Muller, and D. Liepmann, Biomed. Microdevices 3, 225 (2001).

${ }^{16}$ F. Brochard and P. G. de Gennes, J. Chem. Phys. 67, 52 (1977).

${ }^{17}$ J. L. Harden and M. Doi, J. Phys. Chem. 96, 4046 (1992).

${ }^{18}$ M. Fixman, J. Chem. Phys. 69, 1527 (1978).

${ }^{19}$ R. B. Bird, C. F. Curtiss, R. C. Armstrong, and O. Hassager, Dynamics of Polymeric Liquids (Wiley, New York, 1987), Vol. 2.

${ }^{20}$ M. Chopra and R. G. Larson, J. Rheol. 46, 831 (2002).

${ }^{21}$ R. M. Jendrejack, J. J. de Pablo, and M. D. Graham, J. Chem. Phys. 116, 7752 (2002).

${ }^{22}$ H.-C. Öttinger, Stochastic Processes in Polymeric Fluids (Springer, Berlin, 1996).

${ }^{23}$ R. M. Jendrejack, M. D. Graham, and J. J. de Pablo, J. Chem. Phys. 113, 2894 (2000)

${ }^{24}$ C. Pozrikidis, Introduction to Theoretical and Computational Fluid Dynamics (Oxford University Press, New York, 1997).

${ }^{25}$ M. Fixman, Macromolecules 19, 1204 (1986). 
${ }^{26}$ R. M. Jendrejack, J. J. de Pablo, and M. D. Graham, J. Non-Newtonian Fluid Mech. 108, 123 (2002).

${ }^{27}$ J. Rotne and S. Prager, J. Chem. Phys. 50, 4831 (1969).

${ }^{28}$ J. F. Marko and E. D. Siggia, Macromolecules 27, 981 (1994).

${ }^{29}$ J. F. Marko and E. D. Siggia, Macromolecules 28, 8759 (1995).

${ }^{30}$ R. M. Jendrejack, J. J. de Pablo, and M. D. Graham, Technical Proceedings of the Fifth International Conference on Modeling and Simulation of Microsystems (Applied Computational Research Society, San Juan, Puerto Rico, 2002), pp. 88-91.

${ }^{31}$ R. G. Larson, T. T. Perkins, D. E. Smith, and S. Chu, Phys. Rev. E 55, 1794 (1997).

${ }^{32}$ J. S. Hur, E. S. G. Shaqfeh, and R. G. Larson, J. Rheol. 44, 713 (2000).

${ }^{33}$ R. G. Larson, H. Hua, D. E. Smith, and S. Chu, J. Rheol. 43, 267 (1999).

${ }^{34}$ B. Ladoux and P. S. Doyle, Europhys. Lett. 52, 125 (2000).

${ }^{35}$ J. Happel and H. Brenner, Low Reynolds Number Hydrodynamics (Kluwer, Dordrecht, 1991)

${ }^{36}$ C. Pozrikidis, Boundary Integral and Singularity Methods for Linearized Viscous Flow (Cambridge University Press, Cambridge, 1992).
${ }^{37}$ E. R. Dufresne, T. M. Squires, M. P. Brenner, and D. G. Grier, Phys. Rev. Lett. 85, 3317 (2000)

${ }^{38}$ C. K. Colton, C. N. Satterfield, and C.-J. Lai, AIChE J. 21, 289 (1975).

${ }^{39}$ 3D quadratic interpolation functions are used.

${ }^{40}$ B. U. Felderhof, Physica A 89, 373 (1977).

${ }^{41}$ C. W. J. Beenakker, J. Chem. Phys. 85, 1581 (1986).

${ }^{42}$ K. R. Hase and R. L. Powell, Phys. Fluids 13, 32 (2001).

${ }^{43}$ H. Faxen, Arch. Mat. Astron. Fys. 17, 27 (1923).

${ }^{44}$ C. N. Satterfield, C. K. Colton, and W. H. Pitcher, AIChE J. 19, 628 (1973).

${ }^{45}$ D. S. Cannell and F. Rondelez, Macromolecules 13, 1599 (1980).

${ }^{46}$ E. M. Renkin, J. Gen. Physiol. 38, 225 (1953).

${ }^{47}$ J. D. Ferry, J. Gen. Physiol. 20, 95 (1936).

${ }^{48}$ O. B. Bakajin, T. A. J. Duke, C. F. Chou, S. S. Chan, R. H. Austin, and E. C. Cox, Phys. Rev. Lett. 80, 2737 (1998).

${ }^{49}$ We used a quadratic velocity, linear pressure formulation. The resultant sparse, symmetric system of equations was solved using GMRES with a partial LU decomposition preconditioner. 